\title{
The effect of probiotic supplementation on glycemic control and lipid profile in patients with type 2 diabetes: A randomized placebo controlled trial
}

Elham Razmpoosh a, Amir Javadi b, Hanieh Sadat Ejtahed c, d, Parvin Mirmiran a, Maryam Javadi e, f, *, Abbas Yousefinejad g

\begin{abstract}
a Nutrition and Endocrine Research Center, Research Institute for Endocrine Sciences, Shahid Beheshti University of Medical Sciences, Tehran, Iran

b Department of Social Medicine, School of Medicine, Qazvin University of Medical Sciences, Qazvin, Iran c Obesity and Eating Habits Research Center, Endocrinology and Metabolism Molecular-Cellular Sciences Institute, Tehran University of Medical Sciences,

Tehran, Iran

d Endocrinology and Metabolism Research Center, Endocrinology and Metabolism Clinical Sciences Institute, Tehran University of Medical Sciences, Tehran,

Iran

e Children Growth Research Center, Qazvin University of Medical Sciences, Qazvin 34197-59811, Iran

f Department of Nutrition, School of Health, Qazvin University of Medical Sciences, Qazvin 34159-14595, Iran

g Department of Community Nutrition, School of Nutritional Sciences and Dietetics, Tehran University of Medical Sciences, Tehran, Iran
\end{abstract}

\section{A b s t r a c t}

Aims: The role of gut microbiota in the pathogenesis of diabetes is increasing; this study investigates the effect of multi-strain probiotics on fasting plasma glucose (FPG), plasma insulin and lipid profile among patients.

Methods: This randomized double blind controlled trial was performed among 60 patients; individuals were randomly assigned into 2 groups of 30 participants in order to take either probiotic supplements or placebo for 6 weeks. The probiotic supplement consisted of 7 viable strains Lactobacillus, Bifidobacterium and Streptococcus. Nutrient intakes were estimated using a 3-day and 24 hour-dietary recall at the beginning and end of study. Fasting blood samples were taken before and after intervention to measure the levels of FPG, plasma insulin and lipid profiles.

Results: Within group comparisons showed significant decrease and increase in the levels of FPG $\left(\mathrm{P}^{1 / 4}\right.$ 0.001) and HDL-C $\left(\mathrm{P}^{1 / 4}\right.$ 0.002) in probiotic group, respectively. No significant alterations were observed for within and between group comparisons in the levels of insulin, triglycerides, total cholesterol, insulin resistance and anthropometric measurements, including weight, waist circumference and body mass index (all P > 0.05). Conclusions: This study showed a significant decrease in FPG level by multi-strain probiotic supplements in within group comparison; though, further studies are needed to confirm results. (IRCT Code: IRCT2013100714925N1) 